\title{
Proper environment and the SEP account of biological function
}

\author{
Michael Bertrand
}

Received: 21 December 2009 / Accepted: 7 February 2011

(C) Springer Science+Business Media B.V. 2011

\begin{abstract}
The survival enhancing propensity (SEP) account has a crucial role to play in the analysis of proper function. However, a central feature of the account, its specification of the proper environment to which functions are relativized, is seriously underdeveloped. In this paper, I argue that existent accounts of proper environment fail because they either allow too many or too few characters to count as proper functions. While SEP accounts retain their promise, they are unworkable because of their inability to specify this important feature. However, I suggest that this problem can be overcome by the application of a new strategy for specifying proper environment that is grounded in the operation of natural selection and I conclude by offering a first approximation of such an account.
\end{abstract}

Keywords Biological function - Proper function - Serendipitous malfunction · Survival enhancing propensity

\section{Introduction}

Since Wright's $(1973,1976)$ influential work on the subject, etiological or historical accounts of biological function have been widely accepted. Under these accounts, the biological function of a character "is the property it has or had in virtue of which it was promoted by natural selection in the past" (Walsh 1996, p. 533). According to many proponents, the success of etiological accounts come at the expense of their main rival: ahistorical accounts, many of which claim (roughly) that "the biological function of a trait is that property which now contributes to the survival and reproduction of those

M. Bertrand $(\varangle)$

Department of Philosophy, UNC Chapel Hill, Caldwell Hall CB\# 3125, 240 East Cameron,

Chapel Hill, NC 27599-3125, USA

e-mail: michael.d.bertrand@gmail.com 
individuals possessing it, irrespective of what its past tokens have done" (Walsh 1996, p. 533). ${ }^{1}$

I echo Mitchell (1995) and Walsh (1996) in claiming that historical and ahistorical accounts are not incompatible and so these proponents are mistaken. Although I have little to add to the arguments given by these authors, I find it highly plausible that historical and ahistorical accounts do not offer competing analyses but instead offer answers to the following two different questions. As Nancy Cartwright points out, "explanations give answers not only to why questions, but also to what questions. They say of something, what it really is" (1986, p. 203). Like other explanations, functional ascriptions not only explain why a character is manifested but also identify the nature of a character by identifying its functional role in its proper environment. ${ }^{2}$ Thus, "function is used in different scientific projects, there is not a single, univocal explanatory task for which such language is employed in scientific practice" (Mitchell 1995, p. 51).

In this paper, I intend to take advantage of Mitchell and Walsh's insight by exploring what I think is the most promising ahistorical analysis of function available: John Bigelow and Robert Pargetter's SEP account. According to SEP, something has a (biological) function just in case it confers on the organs or organisms that possess it a propensity that is survival enhancing in that organ or organism's proper environment. ${ }^{3}$ While the etiological account may successfully answer why questions, I think that some version of Bigelow and Pargetter's SEP account will provide the best answers to what questions concerning biological functions and as such, it is in our best interest to develop the SEP account.

However, significant development is needed if the SEP account is to deliver on its potential as a way of understanding the nature of function. Although Bigelow and Pargetter identify several places where the need for development is great, I will focus on the specification of the proper environment: the environment to which our judgment concerning whether or not a survival enhancing propensity (sep) is conferred by a character (and so whether a character is properly functioning) is to be relativized. This specification is problematic because of Bigelow and Pargetter's identification of proper environment in terms that "patently involve the notion of proper function" (Plantinga 1993, p. 206): "the natural habitat of the item in question will be a functioning, healthy, interconnected system of organs or parts of the type usual for the species in question" (Bigelow and Pargetter 1987, p. 192). As is indicated by the inclusion of 'healthy,'

\footnotetext{
1 Noteworthy exceptions to this characterization of ahistorical accounts are causal role accounts like those offered by Cummins $(1975,1983)$. These accounts analyze functions in terms of capacities without mention of survival or reproduction. However, there has been some discussion as to whether or not Cummins' account has the resources to analyze proper function (see Millikan 1984; Griffiths 1993). While it would be necessary to address this kind of account in order to fully motivate the survival enhancing propensity (SEP) account of function I will endorse, doing so falls outside the scope of this paper, which is only intended to solve a specific problem with this account.

2 A similar observation is made by Bardon (2007, p. 153). In fact, the ability to answer what questions appears to be just what is meant when proponents of ahistorical accounts claim that their views are forward looking.

3 Bigelow and Pargetter use the term 'natural habitat' while I use 'proper environment' in accordance with more recent work on the subject. I consider these terms to be interchangeable.
} 
Bigelow and Pargetter clearly intend for 'functioning' to mean 'properly functioning' and so proper environment is defined in terms of proper function. Because of this, SEP's use of proper environment in the analysis of function is viciously circular. ${ }^{4}$

Though their stated analysis fails, Bigelow and Pargetter also give several indications of alternate conceptions of proper environment. In my second section, I will briefly discuss the identification of proper environment with current environment. This conception is popular with critics of the SEP account (see for example Walsh 1996; Mossio et al. 2009) and is also suggested by Bigelow and Pargetter when, on p. 192 of Functions, they address the possibility of sudden changes in environment. I will briefly present several objections against this understanding and conclude that we should instead prefer another conception of proper environment.

In my third and fourth sections, I examine a modification to SEP made by Adrian Bardon, the only proponent of the SEP account to address the problem of proper environment. Bardon suggests that a temporal requirement should be incorporated formally into the account. Such a requirement would specify the frequency with which environmental factors must occur and the duration for which an environment must persist in order to be a proper environment. Bigelow and Pargetter also suggest this approach, in the form of an unspecified threshold that an environment must cross before being considered proper. While interesting, I argue that, as a result of the way his temporal requirement is specified, Bardon's account construes proper function too narrowly by excluding characters with intuitive proper functions that are unique to groups smaller than species. Light revisions of Bardon's temporal requirement construe proper function too broadly and so admit intuitive malfunctions. It seems to me that these objections are fatal to his account.

If I am correct, then all existing conceptions of proper environment fail. This is a serious and underappreciated problem for proponents of SEP accounts and, given that SEP accounts are the best way of answering what questions concerning function, this is a problem for any friend of functions. Fortunately, I do not think that this problem is an insurmountable one and, to support this, I will conclude by briefly suggesting a selection-based strategy by which a successful temporal threshold might be developed. According to this strategy, proper environments are those in which selection in favor of the trait in question occurs. I argue that the selection based strategy that I suggest is flexible enough to attribute proper functions to characters manifested in groups of all sizes but is also rigid enough to exclude intuitive cases of malfunction.

\section{Is proper environment identical to current environment?}

It is tempting to generate a straightforward SEP account of function by identifying proper environment with current environment. As I mentioned before, critics of SEP accounts often do this and as these critics point out, it is not a promising approach. I agree with these critics and, in this section, I will give two objections against the current environment interpretation of Bigelow and Pargetter's SEP account. If these objections are not fatal, then they are strong reasons to prefer a new approach.

\footnotetext{
4 Alvin Plantinga gives this argument on p. 206 of his 1993.
} 
The cracks in the current environment interpretation begin to show when we consider what happens immediately after a change in environment. Bigelow and Pargetter note that, in these cases, it will be ambiguous for a period of time which environment we should consider the natural one: is it the environment of the recent past or the one that was only recently occupied by the trait? The current environment interpretation resolves this ambiguity by siding with the recently occupied environment in every case. As I will show, this comes at a high price. Barden notes that "one problem that [Bigelow and Pargetter's] propensity analysis is intended to solve is that of how to distinguish an organism's proper environment from a habitat that, by sheer chance, might be the organism's actual habitat for a period of time" (2007, p. 56).

However by identifying the proper environment with the actual one, the current environment interpretation is unable to solve this problem. As a result, function is relativized to whatever environment an organ or organism finds itself in no matter how short a time this environment is occupied. For example, we are forced to ask ourselves what function bile secretion performs in a liver that has recently been removed by a surgeon from an organ donor for transplant. Once the liver is in the recipient, we must again reconsider the functions of the liver's characters relativized to the environment of its new host. A similar case involving organisms might have us relativizing functions to the chimpanzee enclosure at the zoo or the test cages in a laboratory. It is clear that in the cases I have mentioned, organs or organisms are not in their proper environments but only in environments that they, by chance, occupy for a period of time. ${ }^{5}$

As is indicated by my examples, the common environment interpretation appears to wrongly categorize paradigmatically artificial environments as natural environments. This is so because it seems that natural environment is just a synonym for proper environment. This is indicated by the use of these terms interchangeably in the literature on the SEP account (see footnote 3) and is also borne out by our intuition that characters have proper functions in their natural environments. It would seem that if an organ or organism's current environment is identical to its proper environment in every case, then this distinction between natural and artificial environments breaks down.

However, my argument against the current environment approach does not hang on this point. Intuitively natural examples are commonly available that require organisms to rapidly change environments. For example, Canadian Geese (Branta Canadensis) migrate annually from breeding grounds in northern Canada to as far south as South Carolina, passing through a wide variety of environments in a short period of time. The common environment interpretation requires that we relativize functions in migration cases to every environment an organism passes through no matter how short a time it is occupied. As these organisms progress along their migration route, old environments are replaced as the proper environment as new ones are occupied and function ascriptions must be relativized in each case to the new proper environment. Similarly,

\footnotetext{
5 It might be argued that function ascriptions do not change in relation to every new environment, but instead only change in those environments that are different enough from their successors. In this way, the force of my argument might be blunted. However, it seems that all that is required for an environment to be different enough is for it to impact proper function. As a result, this suggestion becomes trivial. Not surprisingly, it is also of no help to the current environment interpretation because this interpretation just amounts to the claim that the environment an organism is currently in has an impact on proper function.
} 
when a new mammal is born and so moves from the environment provided by its mother to some external environment, the current environment interpretation requires that we reconsider all of the organism's functions by relativizing them to this new environment. ${ }^{6}$ As in cases involving intuitively artificial environments like those above, the current environment interpretation violates our intuitions and fails to reflect the common biological use of function ascriptions by requiring that function ascriptions change far too quickly. This is because it fails to distinguish between proper environments and those environments that an organism just happens to occupy for a period of time.

Because it relativizes functions to environments that are short lived, the current environment approach is also vulnerable to a second objection. In some short-lived environments, an intuitive case of malfunction will confer a sep on an organism. In these cases, intuition and the current environment interpretation part ways to the detriment of the current environment interpretation. To illustrate, imagine a case where a miner is trapped underground in a small chamber. In this environment, which has limited oxygen, pre-existing characters like a very slow heart rate and slow, shallow respiration would confer on the miner a sep by reducing her need for oxygen. Does this make it the case that, in this environment, such weak vital signs indicate that her heart and lungs are functioning properly? I think not. Instead we are inclined to say that the heart and lungs of the miner are malfunctioning, albeit in a lucky way. If the current environment approach admits cases like these, often called cases of serendipitous malfunction, as genuine instances of proper function then it is too permissive. Another interpretation should be found.

\section{SEP*}

In each of my objections above, I argued that the current environment interpretation failed because it countenanced environments that were too short lived. It might be tempting, then, to introduce a temporal requirement that would allow us only to select environments that have persisted for a long enough time. Adrian Bardon suggests just such a modification to Bigelow and Pargetter's SEP account that he calls SEP*:

(SEP*) A character of an organ or organism has an n-function iff: the character generates survival-enhancing propensities for the organism in circumstances that have been common both to the activity of the mechanism that produces the character and to the species to which the character belongs (Bardon 2007, p. 57$){ }^{7}$

This temporal requirement defines proper environment in terms of common circumstances, which is delimited by a two-fold threshold. Common circumstances

\footnotetext{
6 While it seems true that the functions of some characters (like the placenta for example) change under these circumstances, it seems clearly false that the function of every character has the potential to change. For example, this doesn't seem to be a possibility for the functional roles played by the heart or the brain.

7 The ' $n$ ' here stands for naturalistic function in order to distinguish biological functions from common functions, which are those functions that are ascribed to artifacts.
} 
must occur for a long enough duration and with enough frequency to first "result in changes (via accidental mutation and natural selection) to a species' common genetic code" and second to be reflected in the genetic mechanism of the character (Bardon 2007, p. 57). ${ }^{8}$ Call the former condition the 'code threshold condition' and the latter condition the 'mechanism threshold condition' for ease of reference. Characters that generate survival-enhancing propensities in environments that go on to satisfy the temporal requirement have proper functions right from the start and for as long as these environments satisfy the temporal requirement and so are proper environments. However, characters that generate survival enhancing propensities in circumstances that have occurred too infrequently or for too short a duration to satisfy both the code and mechanism threshold conditions and so do not go on to satisfy the threshold requirement are not functions under SEP*. As a result, $\mathrm{SEP}^{*}$ is not vulnerable to either of the objections I urged against the current environment interpretation in Sect. 2.

Even so, there is a significant difficulty with SEP*. Unlike the current environment interpretation, which was too permissive, SEP* is far too restrictive. The problem is the code threshold condition. It is not clear that there is such a thing as the common genetic code of a species. However, I argue in this section that even if this feature of Bardon's account is accepted for the sake of argument, the account fails as it is stated because biologists are not always interested in characters that are shared by an entire species. Sometimes, the character of concern is distinctive to a subspecies or even a subpopulation within a species or subspecies. These kinds of characters confer survival-enhancing propensities evolved in response to selection pressures that are not shared with the rest of the species. The genetic mechanisms of these characters reflect the relevant selection pressures, which act on the subspecies or subpopulation (as the case dictates) but not on the entire species, and so the mechanism threshold condition is fulfilled. However, because the selection pressures in the environment act only on the subspecies or subpopulation and not on the species as a whole, these selection pressures are not reflected in the common genetic code of the species.

While these characters generate survival enhancing propensities, they do not do so in environments that satisfy the two-fold threshold of the temporal requirement and so these characters are excluded from proper function by SEP* even though they are consistent with both the intuitive and common biological use of proper function. In these cases, the circumstances that give rise to the character of concern will never be common to the species. Yet, it seems right to consider characters like these to be instances of proper function even though they fail to satisfy SEP*. So it seems that SEP* is not a genuine analysis of proper function.

To illustrate this point, consider the species Panthera tigris, which is composed of eight geographically isolated subspecies that have unique characters evolved in response to the selection pressures of their significantly different environments. The Panthera tigris sumatrae subspecies, the Sumatran tiger, has closely spaced stripes

\footnotetext{
8 There is a trivial sense of "changes to a species common genetic code" where genetic change in any individual is a change in the species' common genetic code by virtue of that individual's membership in the species. Read this way the code threshold condition allows any serendipitous malfunction to be considered a proper function, a possibility that Bardon attempts to exclude. This sense is not what the author intends and so I disregard it.
} 
that generate a sep by better camouflaging the Sumatran tiger in its dense jungle environment and so increasing its ability to stalk and kill prey (Miquelle and Nath 2007). These stripes have a genetic mechanism that has evolved in response to the unique selection pressures of the dense jungle.

However, because the rest of the Panthera tigris species does not live in the dense jungle and so does not share these selection pressures, they are not reflected in the species' common genetic code and so do not satisfy the code threshold condition. The camouflaging function performed by the Sumatran tiger's closely spaced stripes cannot be considered an instance of proper function under SEP* because, although they generate a sep, they do not do so in an environment that satisfies the two-fold threshold requirement. So they do not do so in a proper environment. This is so in spite of our strong inclination to consider the camouflaging function of the Sumatran tiger's stripes to be an instance of proper function. Because it seems right to consider this camouflaging function to be a proper function and the dense jungle environment to be its proper environment, it seems clear that SEP* is too strong.

A similar example shows that subpopulations must also be countenanced. Consider Dice and Blossom (1937) seminal study, which described significant color variations in several vertebrate populations inhabiting the Tularosa Basin of New Mexico. This environment is significant because it exhibits a high degree of geographical variation over a small area. Within $25 \mathrm{~km}$, the geographical substrate changes from black basaltic lava to white gypsum dunes. Across this range, Dice and Blossom discovered that the dorsal pelages of pocket mice "ranged from nearly pure black to nearly pure white, closely matching the substrate on which the mice were caught" (Hoekstra 2006, p. 227). This substrate matching was also observed in fence lizards and has been strongly linked by several studies to "predation rates by visual avian hunters" (Hoekstra 2006, p. 227).

Populations of these mice differ in their coloration because of selection pressures that encourage them to blend into their differently colored environments. While white mice and black mice share selection pressures of the same kind, they are not to be mistaken as the same selection pressures. Black mice are pressured to take on the color of black basaltic lava while white mice are pressured to take on the color of white gypsum dunes. The differences in the genotypes of each population of mice, as well as differences manifested in the genetic mechanism of the character, reflect these different selection pressures. However, neither the selection pressures encouraging white coloration nor the pressures encouraging black coloration are manifested in the genetic code of the entire species. ${ }^{9}$ Thus while the coloration of these populations of

\footnotetext{
9 It might be pressed that the trait we are interested in is instead a disjunctive one of the form 'black if tending to live on lava and white if tending to live on gypsum'. It is open to us to be interested in this trait, which is more naturally called the substrate-matching coloration trait. However, it seems that any trait can be paired with a related one in such a way and so this should do nothing to reduce our interest in the trait discussed. In addition, there are important differences between this trait and the more narrow black coloration trait. As long as we might be interested only in the black coloration trait and not in related traits like substrate matching coloration, my argument should be persuasive.
} 
pocket mice convey a sep, it does not do so in a proper environment as defined by SEP*. 10, 11

Because biologists are sometimes interested in the functions of characters manifested uniquely by members of a subspecies or by members of a subpopulation, I think that SEP* should be generalized to include both and I will examine a generalization of this kind in my next section. However as an astute referee points out, it might be tempting to consider the pocket mice in the example above as members of different subspecies that are delimited by their color and not as subpopulations of a single species. ${ }^{12}$ However, a subspecies seems to be a collection of phenotypically similar populations and we are only discussing one population. Indeed, subspecies are often defined in contrast to single populations. In addition, the mice in the Tularosa Basin vary clinally, or in a way that is graduated through space. This variation is a smooth one that transitions from a predominance of one phenotype to a predominance of another with vague borders instead of a dramatic hybrid zone between groups (a step cline is not present) and so it would seem that any division into subspecies would be arbitrary in its treatment of intermediate cases. However my arguments hangs only on our desire to attribute functions to characters unique to small sized populations. Because it does not hang on whether small groups like the black pocket mice of the Tularosa Basin are classified as subspecies or subpopulations, any reader who is inclined may substitute 'subspecies' for 'subpopulation' in the pocket mouse example and from here on out.

\footnotetext{
10 Adaptive coloration like the substrate matching manifested in the pocket mouse is a widespread phenomena. It is not unique to this case. It is so wide spread, in fact, that its study has played a formative role in evolutionary biology (see Hoekstra 2006).

11 Bardon's SEP* account relativizes function not only to environment but also to species because it is the species' common genetic code that determines proper environment. This is significant because it makes unrestricted function ascriptions impossible. Under SEP*, we cannot claim that the heart's function is to pump blood. We must instead claim that the heart's function is to pump blood through organisms in proper environment $\mathrm{E}$, which is defined in relation to species $\mathrm{S}$. As a result, many of our questions about functions and appeals to function ascriptions as explanations are underspecified. It might be thought that this provides the basis for a second objection against Bardon, whose theory excludes many of our normal, unqualified, appeals to function. However, Bardon is not alone in relativizing function to species. He inherits this feature from Bigelow and Pargetter's original account, which does so through proper environment in the same way as SEP*. Etiological accounts also relativize their function ascriptions, though not always to species. This can be seen in Karen Neander's influential formulation of the etiological account, which relativizes function to $\mathrm{O}$ and to those organisms that share the ancestors of $\mathrm{O}$.
}

It is the/a proper function of an item (X) of an organism (O) to do that which items of X's type did to contribute to the inclusive fitness of O's ancestors, and which caused the genotype, of which X is the phenotypic expression, to be selected by natural selection. (1991, p. 174).

As a result, I think that relativization to a population is a ubiquitous, though underdiscussed, feature of accounts of function in general and so is not a reason to reject Bardon's account in particular. Instead, Bardon's account fails because function should be relativized to groups that are sometimes smaller than species and this is not possible under Bardon's account.

12 A subspecies is defined by Mayr and Ashlock as "an aggregate of phenotypically similar populations of a species inhabiting a geographic subdivision of the range of that species and differing taxonomically from other populations of that species" (1991, p. 43). 


\section{Can SEP be generalized?}

In order to accommodate the breadth of biologically interesting functions, a natural suggestion is to define the code threshold condition more permissively to include whatever genetic population is relevant to the case. Such a modified code threshold condition might read "a given selection pressure must occur for a long enough duration and with enough frequency to result in changes (via accidental mutation and natural selection) to the common genetic code of the relevant genetic population" and so require that environmental conditions be common to the genetic population exhibiting the mechanism. Call this modification Generalized SEP*. Though it would seem that some version of SEP* lightly modified in this way will provide an adequate analysis of proper function, I will argue in this section that SEP* cannot be fixed just by taking into account more types of groups than species.

As a result of permitting smaller genetic populations to fulfill the code threshold condition, we lose the ability to distinguish between cases of proper function and intuitive cases of serendipitous malfunction and so succumb to the same arguments that SEP* was designed to avoid. As a result, Bardon's population-indexed temporal requirement fails. If we are to understand proper environment, we must do so in some other way. While the code threshold condition is not too strong in this case, Generalized SEP* fails because, like the current environment interpretation, it allows the attribution of proper function to characters that, according to intuition and common biological use, are serendipitously malfunctioning. Because its threshold conditions limit the relevant genetically defined group to species, Bardon's SEP* account insulates itself from such instances of serendipitous malfunction. This is so because as the relevant genetically defined group grows larger, the chance that such a malfunction will fulfill the threshold requirements decreases.

Bardon argues that if the selection pressures producing a serendipitous malfunction reach the two-fold threshold of the temporal requirement under SEP*, the serendipitous malfunction is in actuality an adaptation and so an instance of proper function. This claim is plausible because of the size of the relevant genetically defined group dealt with by SEP*, which requires a long duration and high frequency of selection pressures for change to be affected. Because of the size of the specified population, SEP* has the virtue of restricting the scope of function to changes and environments on appropriate evolutionary timescales (which will vary depending on the species) while justifiably ignoring short lived changes and environments. However if, as has been suggested above, we are forced to modify SEP* such that the relevant group may be significantly smaller than species or subspecies, then this virtue is absent in Generalized SEP*.

It should be noted that the gene pool relevant to a character of concern shrinks as we allow the term to be used in relation to characters found only in subspecies and shrinks even further when we allow the term to be used in relation to characters found only in subpopulations. Therefore, so does the frequency and duration of selection pressures required by the temporal requirement, which is defined in relation to the relevant genetic population. A consequence of reducing the two-fold threshold of the temporal requirement, as we have done in Generalized SEP*, is that many short lived 
selection pressures have the potential to generate characters that are considered to be properly functioning.

By reducing the size of the relevant gene pool, we increase the likelihood of serendipitous malfunctions. In these cases, malfunctions like a heart that beats 15 times a minute, when paired with other malfunctions like elastic holes in the aorta, confer a sep in contrast with either one of these mutations alone. This is true even though it is intuitively clear that neither the heart nor the aorta is functioning properly. ${ }^{13}$ Subpopulations can be very small: it is claimed, for example, that the minimum viable population of houseflies (Musca domestica) is five organisms. It is conceivable, though unlikely, that all of these organisms could exhibit the same serendipitous malfunction as a result of very short-lived environmental conditions (Reed and Bryant 2000). Imagine that, as the result of some strange heritable mutation, the tracheal sacs responsible for respiration in all of the members of a population of seven houseflies are perforated, causing air to leak out when respiration occurs at a normal rate. Imagine also that by some happy coincidence, a second mutation found in only five of these flies causes them to respire much more slowly and shallowly than the other members of their species and so prevents them from losing catastrophic amounts of air through the holes in their tracheal sacs. Among those with the perforated tracheal sac mutation, only those flies that also have the slow and shallow respiration mutation survive to reproduce.

If this case were to obtain, slow and shallow respiration would confer a sep on the population of surviving houseflies just as it was conferred in the slow heartbeat case discussed above and in the miner case discussed in Sect. 2. Furthermore, slow and shallow respiration would satisfy the two-fold temporal requirement because it is reflected in the genetic mechanism that gives rise to it and is, by random mutation and natural selection, part of the common code of the genetic population of which we are concerned, that is, the population of five surviving flies. Therefore, though it is intuitively clear that this character is a serendipitous malfunction and not an adaptation as we usually understand it, slow and shallow respiration is considered an instance of proper function under Generalized SEP*. Surely, though, such serendipitous malfunctions are not instances of proper function no matter what sep they confer. It seems clear in these cases that a character can satisfy Generalized SEP* and yet not be considered, either by intuition or in common biological use, an instance of proper function. So, Generalized SEP* is not a successful analysis of function. It fails because it indexes its temporal requirement to population size and because it allows any population, however small, to qualify.

\section{A promising strategy}

I have argued that both the common environment interpretation of proper environment and Bardon's SEP* account (along with its plausible modifications) fail to provide us

\footnotetext{
13 I echo Plantinga, who first puts forth this style of argument, in claiming that there is no reason to consider malfunctioning mechanisms that happen to work together to be properly functioning mechanisms. It seems clear that a heart that beats 15 times a minute is still malfunctioning no matter what SEP it confers. It is not a function of the heart to behave in this way. The same can be said for any malfunctioning mechanism (see Plantinga 1993, p. 208). Bigelow and Pargetter expound a similar idea (1987, p. 191).
} 
with a satisfactory analysis of proper environment. So, all the existing accounts of proper environment fail. This is bad news both for the friend of SEP accounts and for the friend of functions because it jeopardizes our ability to answer what questions about what a function really is. In this section, I will suggest that SEP accounts can be saved if they apply a new strategy for understanding proper environment. Like Bardon, I think that proper environment should be specified by way of a temporal requirement. However, I reject Bardon's population-based approach in favor of what might be called a selection-based approach. While Bardon suggests that proper environments are those that make their mark on the common genetic code of the species, I suggest that proper environments are those where selection in favor of the trait in question has occurred.

As an example of what a selection-based approach might look like, consider the following.

(SEPS) A character $\mathrm{C}$ of an organ or organism in a population $\mathrm{P}$ has a function iff $\mathrm{C}$ generates survival enhancing propensities, for the organ or organism that has it, in circumstances in which $\mathrm{P}$ persists or will go on to persist for an evolutionarily significant time period. ${ }^{14}$

The crucial piece of this definition is the phrase 'evolutionarily significant time period,' which serves as the temporal requirement that must be met before circumstances can be considered common. Evolutionarily significant time periods have been clearly defined by Paul Griffiths in connection with his Modern History account of function, a variation of the etiological theory, and I think that we can make use of his definition here as well. Griffiths states that an evolutionarily significant time period is "a period such that, given the mutation rate at the loci controlling $\mathrm{T}$ and the population size, [it is likely for] sufficient variants for $\mathrm{T}$ to have occurred to allow significant regressive evolution if the trait was making no contribution to fitness" (Griffiths 1993, p. 417). Regressive evolution can be defined simply as any change to a character that results because it no longer plays an adaptive role. In order for an environment to satisfy the temporal requirement as we have defined it, a character of the same type as $\mathrm{C}$ must be present in population $\mathrm{P}$ in the environment for an evolutionarily significant period of time. However as long as this threshold condition will be met, proper function is awarded to $\mathrm{C}$, in any organism that is a member of $\mathrm{P}$, that is manifested in the environment. This means that, as in other SEP accounts, whether or not $\mathrm{C}$ has a function at some time $\mathrm{t}$ may depend on what happens during an interval after $t$.

It is important to note that SEPS does not require that a character of concern be actively selected for in a given environment. Instead, all that is required is that the character does not undergo regressive evolution. If this were not so, SEPS would not be able to handle problem cases like those where selection occurred in the distant past or where selection occurred for a character that then changed its function over time. On SEPS, a trait need not be selected for in the recent past as long as the trait confers a sep and is manifested in a proper environment, that is, an environment that has occurred or will go on to occur for an evolutionarily significant amount of time. Similarly, SEPS can successfully handle cases where selection for a character occurred and then this

\footnotetext{
14 As Bardon notes, such an account assumes the availability of an adequate definition of natural selection that can be formulated without reference to proper function (see Bardon 2007 p. 58).
} 
character assumed a new function. All that is required in order for the new function of such a character to be proper is for it to confer a sep in a proper environment. If this character loses its function (becomes vestigial) rather than assuming a new function, it fails to generate a sep and so ceases to have a proper function.

It might be thought that cases where traits are linked would pose more of a problem for SEPS. However, I think that these too are easily resolvable. In cases where linked traits both have proper functions, each function must confer a sep in an environment that occurs for an evolutionarily significant amount of time. However in cases where only one trait enhances survival, it is likely that, because they are linked, both traits will persist in the environment for an evolutionarily significant amount of time. Even so, it is not the case that both traits have a function. This is because, by hypothesis, only one of the linked traits confers a sep. Because a sep must be conferred and the temporal requirement must be satisfied for a trait to have a function, SEPS correctly judges that only one linked trait has a function. This is so in spite of the fact that no regressive evolution occurs in either case and so both traits satisfy the temporal requirement.

Unlike the common environment interpretation, which failed because it was unable to distinguish between accidentally inhabited environments and proper environments and so wrongly classified instances of serendipitous malfunctions, the temporal requirement of SEPS only admits long lived circumstances. This lends a greater degree of stabilization to function, which would otherwise have to be reconsidered at each change of environment. Because of this, it deals effectively with cases like the trapped miner whose slow heart rate and respiration confer a sep in a low-oxygen environment. As we would expect, SEPS classifies these cases as malfunctions because the environments in which they generate a sep have not occurred for a long enough duration and with enough frequency to be considered evolutionarily significant.

This is true also of the lucky houseflies in Sect. 4. Although a sep is generated, the circumstances in which it occurs are too short lived. SEPS correctly classifies the lucky flies' mutations as serendipitous malfunctions. However if the slow and steady breathing mutation and the perforated tracheal sac mutation both persisted for an evolutionarily significant amount of time in the fly population, I think that these mutations would count as adaptations all along and not ever as serendipitous malfunctions. This is so because the temporal requirement of SEPS requires that it be likely that regressive evolution would have occurred if these mutations didn't increase fitness. Because adaptations are just those character changes that increase the fitness of populations in their proper environments, apparent serendipitous malfunctions that persist for an evolutionarily significant period of time and so satisfy SEPS seem clearly to be adaptations and not serendipitous malfunctions at all.

It might be thought that, instead of being too permissive, the temporal requirement of SEPS is too strict and so requires time periods that are sometimes too long. However, I think that this objection underestimates the flexibility of SEPS. Like Bardon's account, the temporal requirement of SEPS is relativized to the relevant population $\mathrm{P}$ of which $\mathrm{O}$ is a member. Because organisms like E. coli reproduce quickly, the amount of time that must elapse before regressive evolution is expected in this population will be very short and so even comparatively short periods of time will be evolutionarily significant for these populations. However for organisms like human 
beings with relatively slow rates of reproduction, only long periods of time will be evolutionarily significant. This flexibility also allows SEPS to take into account all of the groups that biologists are interested in and not just species, because it places no restrictions on the size of the population and instead only restricts how long the character must persist in the population.

To see this, lets return to the pocket mouse example but this time lets consider only the black pocket mouse for the sake of simplicity. As Dice and Blossom discovered, the black dorsal pelage of any member of the population of black pocket mice in the Tularosa Basin confer a sep by reducing predation by avian sight hunters. However, in order to be considered a proper function under SEPS, the genetic mechanism behind the black dorsal pelage character must have been present in the population and environment for a long enough duration and with enough frequency that regressive evolution would be likely were it making no contribution to fitness. Given that this threshold will be met, proper function is awarded to any organism in the population that has manifested the character in this environment. This is true also of both ancestors and descendants provided that they manifest the character in the same environment. The time period required depends on specific details concerning the population in question and particularly on the size of the population and on the mutation rate at the loci controlling the trait of interest.

Because of the specificity of the information required by SEPS, it is often quite difficult to determine proper function with certainty. As a helpful reviewer points out, it might be thought that this could form the basis of a pragmatic objection against the account. It is true that even in well-studied cases like the pocket mouse, the genetic mechanisms of characters like dorsal pelage coloration are still not completely understood. This is enough to prevent us from using SEPS to say with certainty whether the black dorsal pelage trait is properly functioning. However, in practice I think that we are willing to settle for less than certainty. In cases like the pocket mouse, we can be reasonably sure that no regressive evolution is occurring (based on the lack of diversity we observe in coloration) without needing to fully understand the genetic mechanisms behind this trait. This is readily observable and should be enough for us to make function ascriptions with confidence. I stand by SEPS as an analysis of function but this analysis shouldn't be seen to have normative implications for biological practice: biologists often rightly assign function with less evidence then SEPS needs to guarantee that these function ascriptions are true. For the same reason, SEPS is not descriptive of the evidence biologists typical gather to support function ascriptions. Instead, I think that SEPS successfully describes the concept that biologists employ when appealing to function ascriptions as answers to what questions.

Because SEPS incorporates a selection-based temporal requirement and even makes use of insights from Griffiths' Modern History account of function, it might be objected that it is an etiological account rather than an ahistorical account and so that I have misrepresented it and its place in the dialectic. As a result, it might be thought that it is no better suited to answer what questions than any other etiological account. While my account does contain some backward looking elements, like those found in the temporal requirement, I think it is clear that SEPS is forward looking in the way that is characteristic of ahistorical accounts and contrasts it with etiological ones. 
The defining feature of ahistorical accounts of function like Bigelow and Pargetter's SEP is their analysis of function in terms of possibly nonexistent future events: future instances of survival by organisms with the character in question in the proper environment. On ahistorical accounts, functions are subjunctive, dispositional properties of individuals. They say of individuals what is likely to happen in the right circumstances as a result of their having a particular character. This defining feature of ahistorical accounts is preserved by SEPS: if a character is to have a function, it must necessarily generate a sep in the proper environment. Where SEPS is backward looking, and so is similar to etiological accounts, is in its specification of the circumstances in which a sep obtains. Though the backward looking elements of SEPS play this crucial role in characterizing it, I see no reason why this would cause proper function to lose its dispositional character as a result. Because SEPS retains the defining feature of ahistorical accounts and does not define function in terms of actual survival as etiological accounts do, I think that SEPS is clearly an ahistorical account. As a result, it is as well suited to answer what questions as any other ahistorical account.

\section{Conclusion}

The SEP account of proper function appears to provide the best ahistorical analysis of function available and so appears to give the best answers to what questions concerning function. However, I have argued that prior specifications of proper environment, a crucial feature of the SEP account, fail because they are either too permissive or too restrictive. The common environment interpretation, favored by critics, caused proper environment to vary wildly and so to admit serendipitous malfunctions. Similarly, Adrian Bardon's population-indexed temporal requirement interpretation excluded groups smaller than species and, when modified to remedy this, also admitted serendipitous malfunctions. This failure poses a significant problem both for proponents of the SEP account, who find themselves unable to provide an account of proper environment, and also for friends of functions, who find themselves unable to analyze function ascriptions as answers to what questions. However, I think that an analysis of proper environment can be found by way of a selection-based temporal requirement. This interpretation of proper environment avoids the problems that afflict prior proposals and so is free of the objections that I raise and, as a result, seems to offer a viable analysis of proper function. ${ }^{15}$

\section{References}

Bardon, A. (2007). Reliabilism, proper function, and serendipitous malfunction. Philosophical Investigations, 30, 45-64.

Bigelow, J., \& Pargetter, R. (1987). Function. The Journal of Philosophy, 84, 181-196.

\footnotetext{
15 I would like to acknowledge the help and guidance of Marc Lange and Joseph LaPorte, both of whom gave extensive and very helpful comments on earlier drafts of this paper. I am also grateful to audiences at UNC Chapel Hill and Hope College for their helpful questions and comments as well as to two anonymous referees provided by Synthese.
} 
Cartwright, N. (1986). Two kinds of teleological explanation. In A. Donagan, A. N. Perovich, Jr., \& M. V. Wedin (Eds.), Human nature and natural knowledge (pp. 201-210). Dordrecht: D. Reidel.

Cummins, R. (1975). Functional analysis. The Journal of Philosophy, 72, 741-765.

Cummins, R. (1983). The nature of psychological explanation. Cambridge, MA: MIT Press.

Dice, L. R., \& Blossom, P. M. (1937). Studies of mammalian ecology in Southwestern North America, with special attention to the colors of desert mammals. Publication Carnegie Institution of Washington, 485, 1-25.

Griffiths, P. E. (1993). Functional analysis and proper function. The British Journal for Philosophy of Science, 44, 409-422.

Hoekstra, H. E. (2006). Genetics, development and evolution of adaptive pigmentation in vertebrates. Heredity, 97, 222-234.

Mayr, E., \& Ashlock, P. D. (1991). Principles of systematic zoology. New York: McGraw-Hill.

Millikan, R. (1984). Language, thought, and other biological categories: New foundations for realism. Cambridge, MA: MIT Press.

Miquelle, D. G., \& Nath, L. (2007). Tiger. In D. W. Macdonald (Ed.), The encyclopedia of mammals. Oxford: Oxford University Press. Retrieved September 27, 2008, from Oxford Reference Online.

Mitchell, S. D. (1995). Function, fitness, and disposition. Biology and Philosophy, 10, 39-54.

Mossio, M., et al. (2009). An organizational account of biological functions. British Journal of Philosophy of Science, 60, 813-841.

Neander, K. (1991). Functions as selected effects: The conceptual analyst's defense. Philosophy of Science, 58, 168-184.

Plantinga, A. (1993). Warrant and proper function. Oxford: Oxford University Press.

Reed, D. H., \& Bryant, E. H. (2000). Experimental tests of minimum viable population size. Animal Conservation, 3, 7-14.

Walsh, D. M. (1996). Fitness and function. The British Journal for the Philosophy of Science, 47, $553-574$.

Wright, L. (1973). Functions. Philosophical Review, 82, 139-168.

Wright, L. (1976). Teleological explanations: An etiological analysis of goals and functions. Berkeley: University of California Press. 